\title{
The Properties of Isolated Main Galaxies in Sloan Digital Sky Survey Data Release 3 (SDSS DR3)
}

\author{
Xinfa Deng ${ }^{\mathrm{A}, \mathrm{B}}$, Xinsheng Ma ${ }^{\mathrm{A}}$, Chenghong Luo ${ }^{\mathrm{A}}$, Qun Zhang ${ }^{\mathrm{A}}$, Qing-Hua Liao ${ }^{\mathrm{A}}$ and \\ Ji-zhou $\mathrm{He}^{\mathrm{A}}$ \\ A Mathematics and Physics College, Nanchang University, Jiangxi 330047, China \\ B Corresponding author. E-mail: xinfadeng@163.com
}

Received 2005 June 22, accepted 2005 October 7

\begin{abstract}
We have constructed a Main galaxy subsample of 67777 galaxies with redshifts in the range $0.08 \leq z \leq 0.12$ from the Sloan Digital Sky Survey Data Release 3. Using cluster analysis, two isolated Main galaxy samples were extracted from this subsample. The two isolated Main galaxy samples identified at different radii have the same properties. Additionally, we find fewer early-type galaxies in isolated Main galaxy samples than in a close double galaxy sample.
\end{abstract}

Keywords: cosmology — galaxy — cluster analysis

\section{Introduction}

Isolated galaxies, which may have experienced no major interactions in billions of years, are a group of special and rare galaxies in the universe. They are important because they probe regions with the lowest galaxy number densities. They can serve as interesting samples for detecting and studying the properties of galaxies, such as in, for example, studies of the effects of environment on galaxy morphologies and star formation rates (Adams, Jensen, \& Stocke 1980; Haynes \& Giovanelli 1980; Haynes, Giovanelli, \& Chincarini 1984; Koopmann \& Kenney 1998). In recent years, many properties of isolated galaxies have been extensively explored (Aars 2003; Pisano \& Wilcots 2003; Sauty et al. 2003; Stocke et al. 2004; Varela et al. 2004; Marcum, Aars, \& Fanelli 2004).

According to Karachentseva's selection algorithm (Karachentseva 1973), a galaxy $i$ with angular diameter $a_{i}$ is considered isolated if the projected sky separation $x_{i, j}$ between this galaxy and any neighboring galaxy $j$ of angular diameter $a_{j}$ satisfies the following two criteria: $x_{i, j} \geq 20 a_{j}$ and $a_{j} / 4 \leq a_{i} \leq 4 a_{j}$. Using Zwicky's Catalogue of Galaxies and Clusters of Galaxies (Zwicky, Herzog, \& Wild 1968), Karachentseva compiled her isolated galaxy catalogue from work with prints of the Palomar Sky Survey. The original catalogue contained 1052 candidate isolated galaxies, later reduced to 893 galaxies (Karachentseva 1980).

Allam et al. (2005) implemented a variation on the original Karachentseva criteria, and identified isolated galaxies in the Sloan Digital Sky Survey Data Release 1 (SDSS DR1). According to their modified criteria, a galaxy $i$ with a $g$-band magnitude $g_{i}$ and $g$-band Petrosian radius $R_{i}$ is considered to be isolated if the projected sky separation between this galaxy and any neighboring galaxy $j$ satisfies $x_{i, j} \geq 40 R_{j}$ and $\left|g_{i}-g_{j}\right|>$ 3.0. After all rejections and verifications, 2980 isolated galaxies were extracted from imaged sky coverage of approximately $2099 \mathrm{deg}^{2}$.

The above methods of identifying isolated galaxies are based mainly on the two-dimensional projected sky separation and galaxy diameter. The selected galaxies may not be the most isolated in the three-dimensional space. When using a catalogue of galaxies with redshift (a three-dimensional galaxy sample), identifying isolated galaxies only by the projected separation and diameter criteria is less satisfactory. In this paper, we use three-dimensional cluster analysis (Einasto et al. 1984) and extract isolated Main galaxies from SDSS Data Release 3 (SDSS DR3; Abazajian et al. 2005). By cluster analysis, the sample can be separated into isolated galaxies, close double and multiple galaxies, galaxy groups or clusters. At larger radii, most galaxies of the sample form different groups or clusters; few galaxies are isolated. However, these isolated galaxies provide a good sample for studies of three-dimensionally isolated galaxies.

\section{Data}

The Sloan Digital Sky Survey (SDSS) is one of the largest astronomical surveys to date. The completed survey will cover about $10000 \mathrm{deg}^{2}$. York et al. (2000) provided the technical summary of the SDSS. The SDSS observes galaxies in five photometric bands $(u, g, r, i$, and $z$ ) centred at 3540, 4770, 6230, 7630, and $9130 \AA$. The imaging camera was described by Gunn et al. (1998), the photometric system was roughly described in Fukugita et al. (1996), and the photometric calibration of the SDSS imaging data was described in Hogg et al. (2001), and Smith et al. (2002). Pier et al. (2003) 
described the methods and algorithms involved in the astrometric calibration of the survey, and presented a detailed analysis of the accuracy achieved. Many of the survey properties were discussed in detail in the Early Data Release paper (Stoughton et al. 2002). Galaxy spectroscopic target selection can be implemented by two algorithms. The primary sample (Strauss et al. 2002), referred to here as the Main sample, targets galaxies brighter than $r<17.77$ ( $r$-band apparent Petrosian magnitude). The surface density of such galaxies is $\sim 90$ per square degree. This sample has a median redshift of 0.10 and few galaxies beyond $z=$ 0.25. The Luminous Red Galaxy (LRG) algorithm (Eisenstein et al. 2001) selects $\sim 12$ additional galaxies per square degree, using colour-magnitude cuts in $g, r$, and $i$ to select galaxies to $r<19.5$ that are likely to be luminous early-types at redshifts up to $\sim 0.5$.

The SDSS has adopted a modified form of the Petrosian (1976) system for galaxy photometry, which is designed to measure a constant fraction of the total light independent of the surface-brightness limit. The Petrosian radius $r_{\mathrm{P}}$ is defined to be the radius where the local surface-brightness averaged in an annulus equals $20 \%$ of the mean surface-brightness interior to this annulus, i.e.

$$
\frac{\int_{0.8 r_{p}}^{1.25 r_{p}} \mathrm{~d} r 2 \pi r I(r) /\left[\pi\left(1.25^{2}-0.8^{2}\right) r^{2}\right]}{\int_{0}^{r_{p}} \mathrm{~d} r 2 \pi r I(r) /\left[\pi r^{2}\right]}=0.2
$$

where $I(r)$ is the azimuthally averaged surface-brightness profile. The Petrosian flux $F_{\mathrm{P}}$ is then defined as the total flux within a radius of $2 r_{p}, F_{p}=\int_{0}^{2 r_{P}} 2 \pi r \mathrm{~d} r I(r)$. With this definition, the Petrosian flux (magnitude) is $\sim 98 \%$ of the total flux for an exponential profile and $\sim 80 \%$ for a de Vaucouleurs profile. The other two Petrosian radii listed in the photo output, $R_{50}$ and $R_{90}$, are the radii enclosing $50 \%$ and $90 \%$ of the Petrosian flux, respectively.

The SDSS sky coverage can be separated into three regions. Two are located in the north of the Galactic plane, with one region at the celestial equator and another at high declination. The third lies in the south of the Galactic plane, comprised of a set of three strips near the equator. Each of these regions covers a wide range of survey longitude.

In this paper, we consider the Main galaxy sample. The data was downloaded from the Catalog Archive Server of SDSS Data Release 3 (Abazajian et al. 2005) by the SDSS SQL Search (with SDSS flag: bestPrimtarget $=64$, high-confidence redshifts: SZwarning $\neq 16$ and Zstatus $\neq 0,1$ and redshift confidence level: $z$ conf $>0.95$ ) (http:// www.sdss.org/dr3/access/index.html\#CAS). From this sample, we select 214795 Main galaxies in the redshift region: $0.02 \leq z \leq 0.2$.

In calculating distances we use a cosmological model with matter density $\Omega_{0}=0.3$, cosmological constant

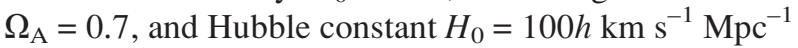
with $h=0.7$.

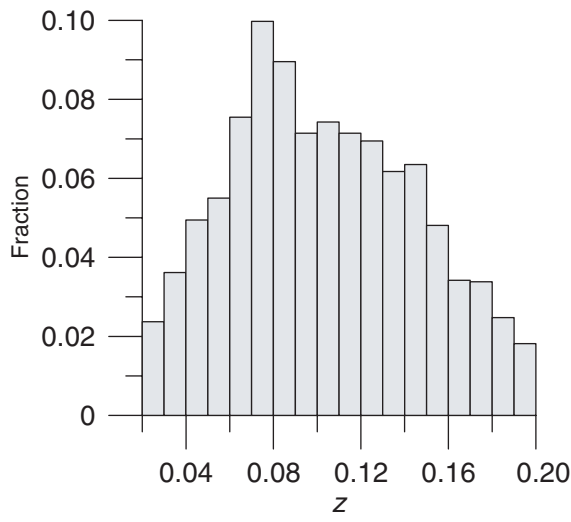

Figure 1 The distribution of redshift $z$ of galaxies for the Main galaxy sample.

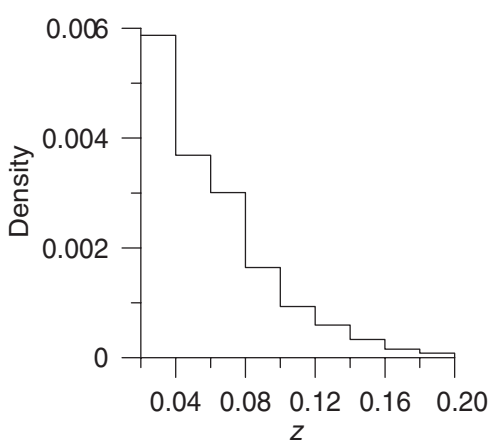

Figure 2 The distribution of space density of galaxies with redshift $z$ for the Main galaxy sample.

Figure 1 illustrates the distribution of redshift $z$ of galaxies for our Main galaxy sample. The peak of the distribution is at about $z=0.08$. Because the Main galaxy sample is an apparent-magnitude limited sample, the space density of galaxies apparently decreases with increasing redshift $z$, as shown in Figure 2. This kind of incompleteness of the sample will influence the results when identifying isolated galaxies. More isolated galaxies will be identified in low density regions than in regions of high galaxy number density. In order to decrease the effect of the radial selection function, we have constructed a Main galaxy subsample (MGSU) in the redshift region $0.08 \leq z \leq 0.12$ which contains 67777 galaxies.

\section{Cluster Analysis}

Cluster analysis (Einasto et al. 1984), as a general method, has been widely applied to studies of the geometry of point samples in many fields. The key to this method is to separate the sample into individual systems by an objective, automatic procedure. Let us draw a sphere of radius $R$ around each sample point (in our case, a galaxy). If there are other galaxies within this sphere, they are considered as belonging to the same system. Call these neighbouring close galaxies 'friends'. Now draw spheres around each new neighbour and 
continue the procedure using the rule 'any friend of my friend is my friend'. When no more new neighbours or 'friends' can be added, the procedure stops and a system is identified. As a result, each system consists of either a single, isolated galaxy or multiple galaxies in which each have at least one neighbour within a distance not exceeding $R$. The resulting number of nominally isolated galaxies decreases with increasing radius. In our analysis, we set the threshold such that the number of isolated galaxies is below $10 \%$ of the total galaxy number in the sample as the condition to identify an isolated galaxy sample.

The mean density of galaxies is $\bar{\rho}=N / V$ (where $N$ is the number of galaxies contained in the volume $V$ ). The radius of the sphere with unit population is $R_{0}=(3 / 4 \pi \bar{\rho})^{-1 / 3}$. For the MGSU, $R_{0}$ is $5.7863 \mathrm{Mpc}$. In our analysis, all distances are expressed as a dimensionless radius $r=R / R_{0}$.

\section{The Basic Properties of Isolated Main Galaxies}

When identifying isolated galaxies by cluster analysis, it is important to realize that there is no a priori defined neighbourhood radius. This forces us to consider and analyse a certain range of neighbourhood radii, and accordingly the properties of systems that form our sample. At small radii only close double and multiple galaxies, cores of groups and conventional clusters of galaxies will form systems, with most galaxies being identified as isolated single galaxies. With an increasing neighbourhood radius, less dense peripherical regions of groups and clusters will be included into systems. At still larger radii neighbouring systems merge into large, usually elongated units; few galaxies are isolated. Figures 3 and 4, respectively, show how the number of isolated single galaxies $\left(N_{\sin }\right)$ and the galaxy number of the richest system $\left(N_{\max }\right)$ change with increasing dimensionless radius $r$ for the MGSU. Figure 4 demonstrates that at radius $r=0.9$, the galaxy number of the richest system is 2687, with a sharp increase evident at $r$ $=0.8$, where $N_{\max }=244$. At this radius, most galaxies of the sample begin to merge into less dense regions of groups and clusters, and a large and less dense system is formed. At radius $r=1.2$, the galaxy number of the richest system reaches 7355 and the number of isolated galaxies is 4099. Figure 3 shows that at this radius the change of number $N_{\sin }$ of isolated single galaxies gradually becomes even smaller as the radius $r$ increases. Isolated galaxies identified at radii $r \geq 1.2$ can be defined as genuinely isolated in three-dimensional space. We select the galaxies identified as isolated at radii $r=1.2$ and $r=1.4$ as two isolated galaxy samples: Sample1.2 and Sample1.4, respectively. Sample1.2 includes 4099 isolated galaxies $(6.0 \%$ of the total galaxy number in the MGSU) and Sample1.4 includes 2596 isolated galaxies (3.8\% of the total galaxy number in the MGSU).

Luminosity, size, and morphological type are amongst the most basic properties of a galaxy. Observed galaxies cover large ranges in these properties, with

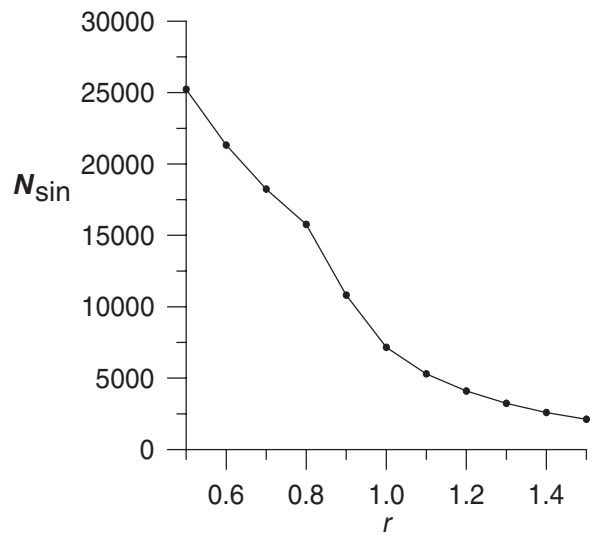

Figure 3 Isolated galaxy number $N_{\text {sin }}$ versus dimensionless radius $r$.

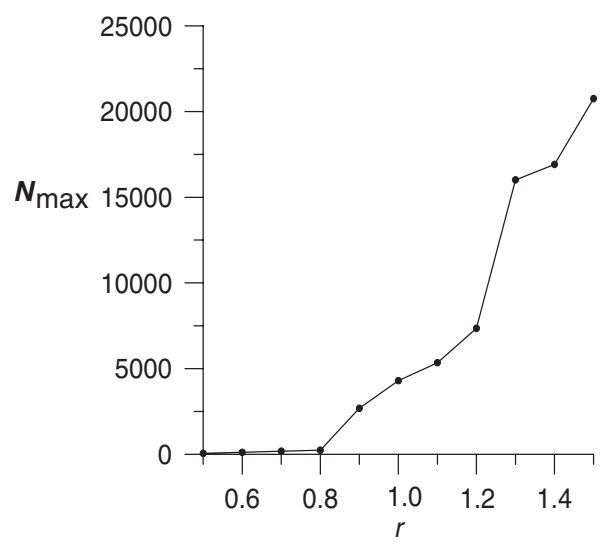

Figure 4 The galaxy number $N_{\max }$ of the richest system versus dimensionless radius $r$.

effective radii between $0.1 \mathrm{kpc}$ and $50 \mathrm{kpc}$ and morphologies changing from pure disk systems to pure ellipsoidal systems. Clearly, studies of the distribution of galaxies with respect to these properties are crucial to our understanding of the formation and evolution of galaxy populations.

Figure 5 shows histograms of the distribution of the luminosities ( $r$-band absolute magnitude, $M_{r}$ ) of galaxies in the MGSU, in Sample1.2 and in Sample1.4. For galaxies at $z<0.12$, the $K$-corrections are trivial and have been ignored (Blanton et al. 2002). The absolute magnitude $M_{r}$ is calculated from the $r$-band apparent magnitude $m_{r}$ as follows: $M_{r}=m_{r}-5 \log D_{\mathrm{L}}-25$, where $D_{\mathrm{L}}$ is the luminosity distance. The distribution of the luminosities of galaxies in the MGSU, in Sample1.2 and Sample1.4 are found to be almost the same.

We select the $r$-band radius $R_{50}\left(R_{50, r}\right)$ as the parameter of galaxy size, and have calculated the concentration index $c i=R_{90} / R_{50}$ which can be used to separate early-type (E, S0) galaxies from late-type (Sa, Sb, Sc, Irr) galaxies (Shimasaku et al. 2001). Using 1500 galaxies with eyeball classification, Nakamura et al. (2003) confirmed that $c i=2.86$ divides visually classified galaxies at $\mathrm{S} 0 / \mathrm{a}$ with a completeness of $\sim 0.82$ for samples of both late and early 


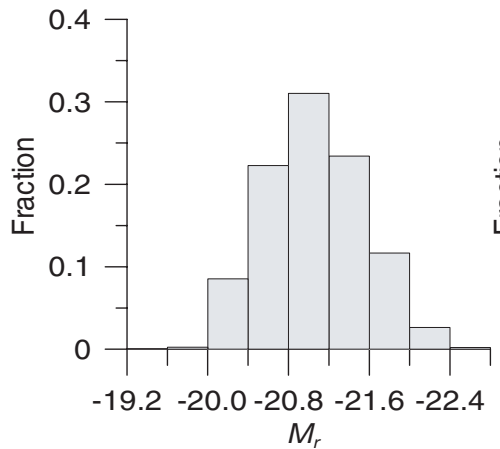

(a)

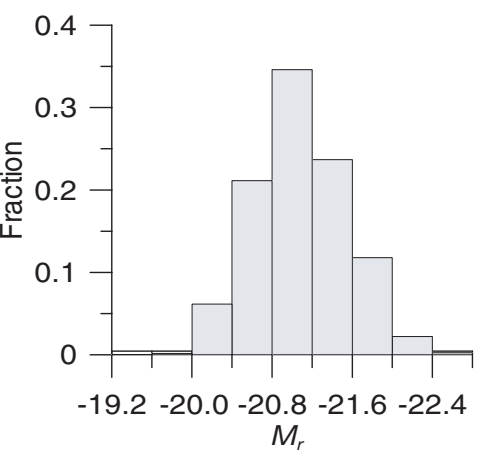

(b)

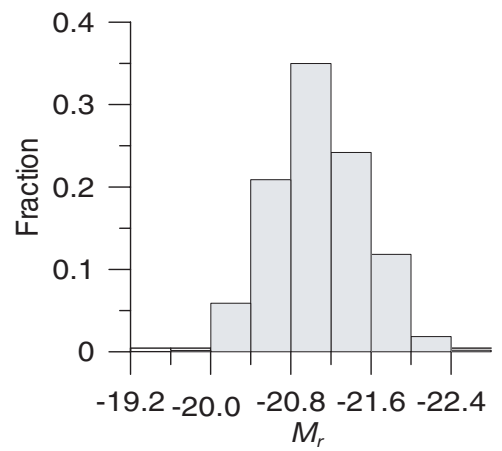

(c)

Figure 5 Histograms of the distribution of luminosities $M_{r}$ of galaxies in (a) the MGSU, (b) Sample1.2, (c) Sample1.4.

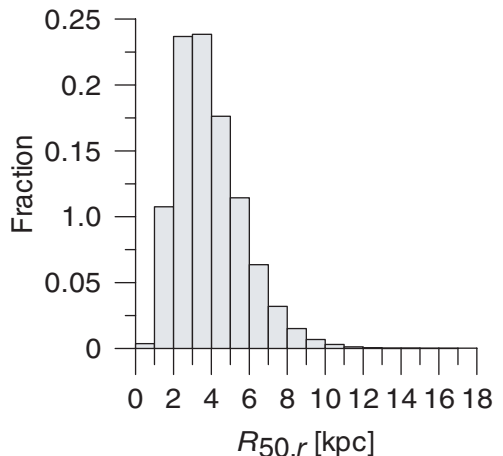

(a)

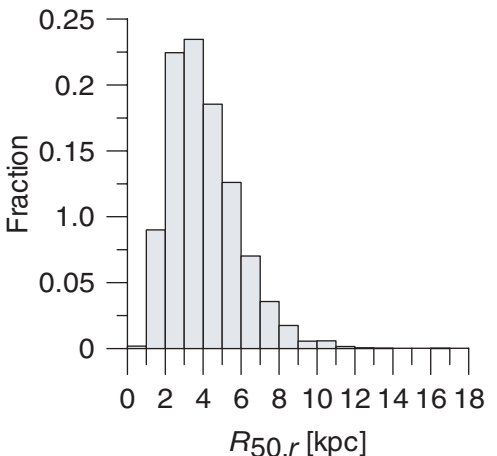

(b)

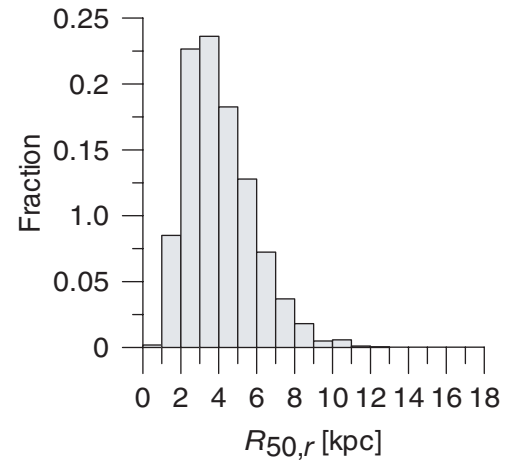

(c)

Figure 6 Histograms of the size distribution of galaxies in (a) the MGSU, (b) Sample1.2, (c) Sample1.4.

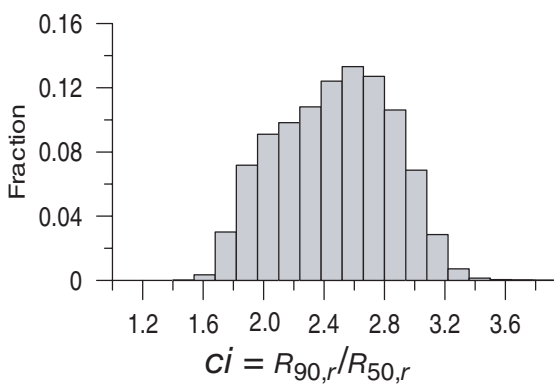

(a)

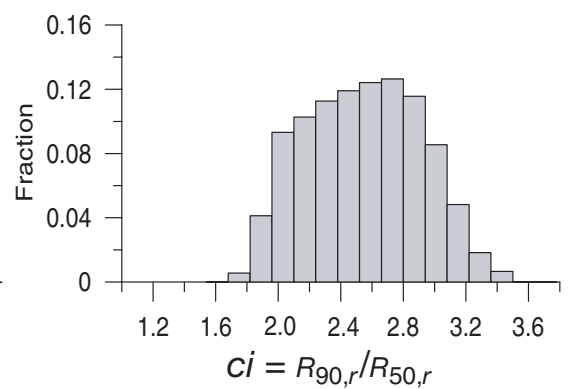

(b)

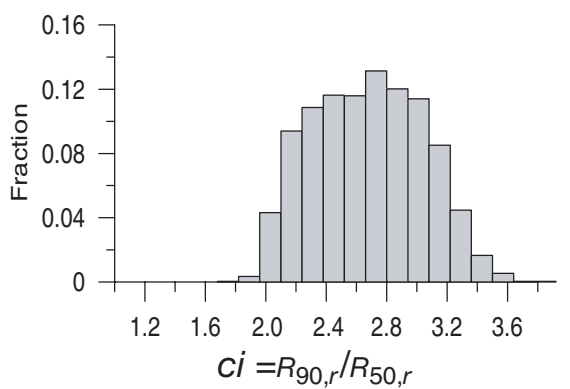

(c)

Figure 7 Histograms of the concentration index ci distribution of galaxies in (a) the MGSU, (b) Sample1.2, (c) Sample1.4.

types. Figures 6 and 7 show, respectively, the size distribution and the concentration index $c i$ distribution of galaxies in the MGSU, Sample1.2, and Sample1.4. In Sample1.2, 22.7\% galaxies have a concentration index $c i>2.86$, and in Sample1.4 this proportion is $22.1 \%$, lower than the proportion $(28.3 \%)$ in the MGSU. This demonstrates that there are fewer early-type galaxies in isolated galaxy samples.

According to the above analysis, we find that isolated galaxy samples identified at two different radii have the same properties. This demonstrates that isolated galaxies identified at these radii by cluster analysis have definite basic properties and can be considered good, isolated galaxy samples.
At very small radii only close double and multiple galaxies will form systems. Moreover, most systems are close double galaxies. In order to compile a real threedimensional galaxy pair catalogue, Deng et al. (2005) extracted close double Main galaxies from SDSS DR3 by cluster analysis. They selected the range of projected separation criteria of most pair samples as the analysis range of neighbourhood radii, and analysed the clustering properties of the Main galaxy sample in the neighbourhood radius range $R=60-200 \mathrm{kpc}$, in order to find a neighbourhood radius which identified galaxy pairs. Further, the distribution of the $r$-band radius $R_{90}$ $\left(R_{90, r}\right)$ of all galaxies for the Main galaxy sample was analysed. They concluded that a neighbourhood radius 


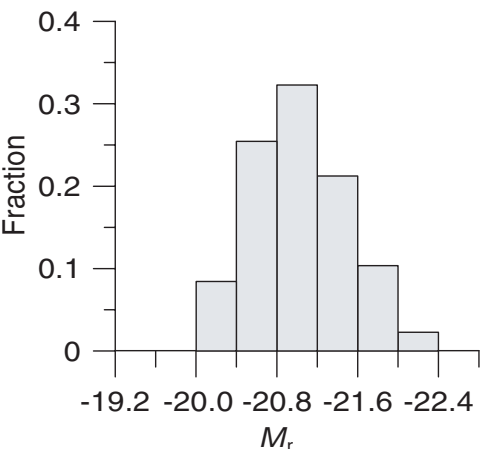

(a)

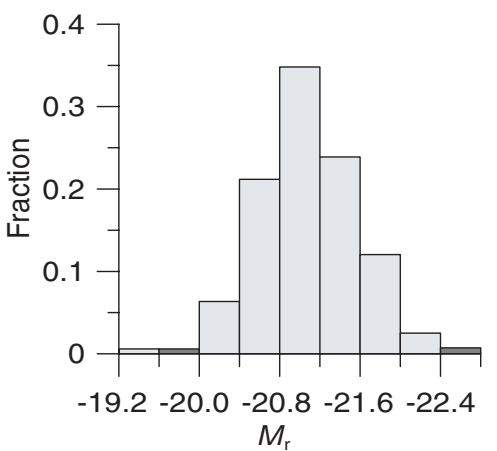

(b)

Figure 8 Histograms of the luminosity distribution of galaxies for (a) galaxy pairs identified at a neighbourhood radius of $R=100 \mathrm{kpc}$ and (b) isolated galaxies identified at dimensionless radius $r=1.2$ (Sample1.2).

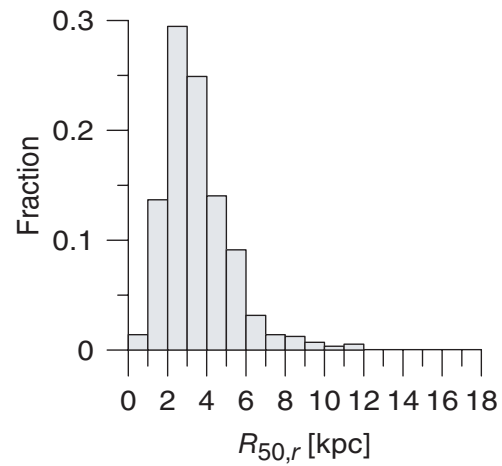

(a)

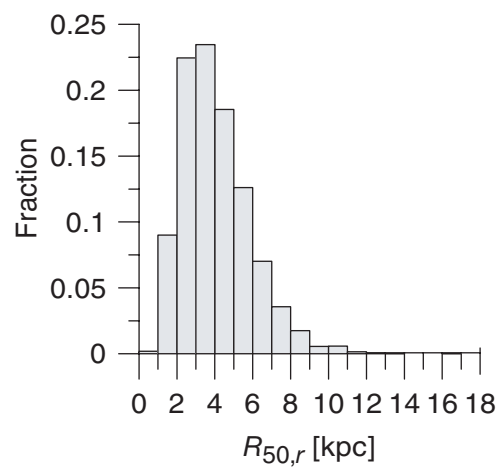

(b)

Figure 9 Histograms of the size distribution of galaxies for (a) galaxy pairs identified at a neighbourhood radius of $R=100 \mathrm{kpc}$ and (b) isolated galaxies identified at dimensionless radius $r=1.2$ (Sample1.2).

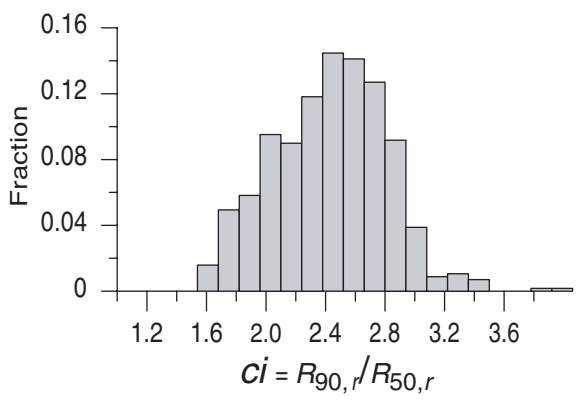

(a)

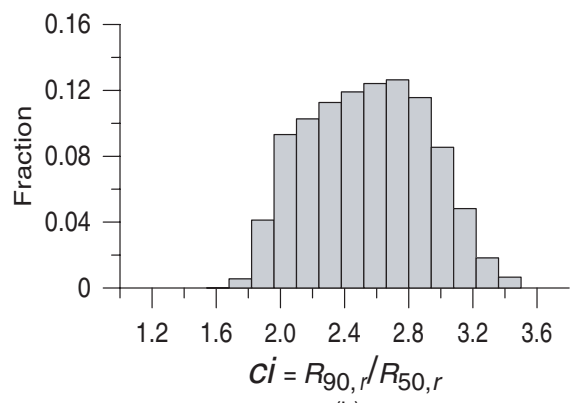

(b)

Figure 10 Histograms of the concentration index $c i$ distribution of galaxies for (a) galaxy pairs identified at a neighbourhood radius of $R=100 \mathrm{kpc}$ and (b) isolated galaxies identified at dimensionless radius $r=1.2$ (Sample1.2).

of $R \approx 100 \mathrm{kpc}$ can be defined as the radius which identifies close galaxy pairs. At a neighbourhood radius of $100 \mathrm{kpc}$, we identify 570 close double galaxies in the MGSU $(0.84 \%$ of the total galaxy number in the MGSU) and select these close double galaxies as our galaxy pair sample. In Figures 8, 9, and 10, we compare the luminosity $M_{r}$, size, and concentration index $c i$ distributions of galaxies of this pair sample with those of the isolated galaxy sample identified at the dimensionless radius $r=1.2$ (Sample1.2). We find that there are more early-type galaxies $(c i>2.86)$ in the galaxy pair sample than in Sample1.2. In the isolated galaxy sample, $22.7 \%$ of galaxies have a concentration index $\mathrm{ci}>$ 2.86 , while in the pair sample the percentage is $37.4 \%$. Additionally, we notice that on average there is a higher proportion of large galaxies (larger than $4 \mathrm{kpc}$ ) in the isolated galaxy sample.

\section{Summary}

In this paper, we identify isolated Main galaxies by cluster analysis, and study the properties of isolated Main galaxy samples identified at different radii. In 
order to decrease the effect of the radial selection function, we have constructed a subsample in the redshift region $0.08 \leq z \leq 0.12$, and refer to this subsample of 67777 galaxies as the MGSU. We set the threshold that the number of isolated galaxy be below $10 \%$ of the total galaxy number in the sample as the condition of identifying an isolated galaxy sample. Two isolated Main galaxy samples are analysed: Sample1.2, identified at dimensionless radius $r=$ 1.2, which includes 4099 galaxies $(6.0 \%$ of the total galaxy number in the MGSU) and Sample1.4, identified at dimensionless radius $r=1.4$, which includes 2596 galaxies (3.8\% of the total galaxy number in the MGSU). Isolated Main galaxy samples identified at different radii are found to have the same properties. This demonstrates that our cluster analysis and the threshold setting are effective in the process of identifying isolated galaxies; the isolated galaxy sample identified below the threshold is a reliable one. Additionally, we find that there are fewer early-type galaxies in isolated Main galaxy samples than in the close double galaxy sample.

\section{Acknowledgements}

Supported by the National Science Foundation of China (10465003).

\section{References}

Aars, C. E. 2003, AAS, 203, 65.01

Abazajian, K. et al. 2005, AJ, 129, 1755

Adams, M., Jensen, E. \& Stocke, J. 1980, AJ, 85, 1010

Allam, S. S., Tucker, D. L., Lee, B. C. \& Smith, J. A. 2005, AJ, 129,2062

Blanton, M. R. et al. 2003, AJ, 125, 2348
Deng, X. F. et al. 2005, Ap\&SS, submitted

Einasto, J., Klypin, A. A., Saar, E. \& Shandarin, S. F. 1984 MNRAS, 206, 529

Eisenstein, D. J. et al. 2001, AJ, 122, 2267

Fukugita, M., Ichikawa, T., Gunn, J. E., Doi, M., Shimasaku, K. \& Schneider, D. P. 1996, AJ, 111, 1748

Gunn, J. E. et al. 1998, AJ, 116, 3040

Haynes, M. P. \& Giovanelli, R. 1980, ApJ, 240, L87

Haynes, M. P., Giovanelli, R. \& Chincarini, G. L. 1984, ARA\&A, 22,445

Hogg, D. W., Finkbeiner, D. P., Schlegel, D. J. \& Gunn, J. E. 2001, AJ, 122, 2129

Karachentseva, V. E. 1973, SoSAo, 8, 3

Karachentseva, V. E. 1980, AZh, 57, 1153

Koopmann, R. A. \& Kenney, J. D. P. 1998, ApJ, 497, L75

Marcum, P. M., Aars, C. E. \& Fanelli, M. N. 2004, AJ, 127, 3213

Nakamura, O., Fukugita, M., Yasuda, N., Loveday, J., Brinkmann, J., Schneider, D. P., Shimasaku, K. \& SubbaRao, M. 2003, AJ, 125,1682

Petrosian, V. 1976, ApJ, 209, L1

Pier, J. R., Munn, J.A., Hindsley, R. B., Hennessy, G. S., Kent, S. M., Lupton, R. H. \& Ivezić, Z. 2003, AJ, 125, 1559

Pisano, D. \& Wilcots, E. M. 2003, ApJ, 584, 228

Sauty, S. et al. 2003, A\&A, 411, 381

Shimasaku, K. et al. 2001, AJ, 122, 1238

Smith, J.A. et al. 2002, AJ, 123, 2121

Stocke, J. T., Keeney, B. A., Lewis, A. D., Epps, H. W. \& Schild, R. E. 2004, AJ, 127, 1336

Stoughton, C. et al. 2002, AJ, 123, 485

Strauss, M.A. et al. 2002, AJ, 124, 1810

Varela, J., Moles, M., Márquez, I., Galleta, G., Masegosa, J. \& Bettoni, D. 2004, A\&A, 420, 873

York, D. et al. 2000, AJ, 120, 1579

Zwicky, F., Herzog, E. \& Wild, P. 1968, Catalogue of Galaxies and of Clusters of Galaxies (Pasadena: California Institute of Technology) 